\title{
Some Remarks on the Padé Unitarization of Low-Energy Amplitudes
}

\author{
P. Masjuan, ${ }^{a}$ J.J. SAnZ-Cillero $^{a}$ and J. Virto ${ }^{b}$ \\ ${ }^{a}$ IFAE, Universitat Autònoma de Barcelona, 08193 Bellaterra, Barcelona, Spain \\ ${ }^{b}$ Università di Roma "La Sapienza" and INFN Sezione di Roma, 00185 Roma, Italy
}

May 21, 2008

\begin{abstract}
We present a critical analysis of Padé-based methods for the unitarization of low energy amplitudes. We show that the use of certain Padé Approximants to describe the resonance region may lead to inaccurate determinations. In particular, we find that in the Linear Sigma Model the unitarization of the low energy amplitude through the inverse amplitude method produces essentially incorrect results for the mass and width of the sigma. Alternative sequences of Padés are studied and we find that the diagonal sequences (i.e., $[N / N]$ ) have much better convergence properties.
\end{abstract}

\section{Introduction}

Effective Field Theories (EFT) have become a very useful tool for the description of low-energy physics [1]. Based on symmetry and dimensional analysis arguments, they allow to organize a perturbative expansion of the amplitudes in powers of soft momenta over some characteristic scale, $p / \Lambda$. For instance, below the lightest resonance multiplet, the interactions between the pseudoNambu-Goldstone bosons from the spontaneous chiral symmetry breaking -the pions in the $S U(2)$ case- is provided by Chiral Perturbation Theory $(\chi \mathrm{PT})$ [2, 3, 4]. Its range of validity is a few hundred $\mathrm{MeV}$, breaking down as one approaches new states not included in the EFT. Thus, $\chi$ PT is unable to describe the resonance region and one needs to incorporate additional ingredients.

Several works have tried to extend the range of validity of the EFT by means of the unitarization of the low-energy amplitude. Unitarization methods have been applied extensively to Quantum Chromodynamics and $\chi \mathrm{PT}$ [5], where the issue of the scalar resonances is particularly interesting. However, their range of applicability is wider; for example they have also been applied to $W W-$ scattering [6]. Padé Approximants (PAs) [7, 8] and the Inverse Amplitude Method (IAM) [9, 10] are among the most usual ones, although some remarks and criticisms on the reliability of these and other unitarization methods have been raised [8, 11, 12]. In the present letter we focus our attention 
on the PAs. We study up to what point one can rely on them to describe the resonant properties of the theory. By means of a couple of models (the Linear Sigma Model and a vector resonance model), we show that these unitarization procedures may lead to improper determinations of the resonance pole position (masses and widths) [13]. Furthermore, one may not recover the right values for the Low-Energy Couplings (LECs) of the EFT if the PAs are applied to describe the resonant region.

The starting point of our analysis is, therefore, a model where the properties of the resonances are known. Then we derive and unitarize the corresponding low-energy amplitude. The predictions for the masses and widths obtained from the PA sequence $[1 / N]$ (referred to as IAM in some works [8, 9, 10, 12]) are compared and found to be quite different from those of the original model. As an alternative to the badly behaved sequence $[1 / N]$, we propose the use of the PA sequence $[N / N]$, which quickly converges as $N$ is increased. For simplicity the chiral limit will be assumed all along the paper, but this will not alter the main conclusion.

The structure of this letter is the following. We begin, in Section 2, with the analysis in the LSM: in Section 2.1 we compute the exact position of the pole of the sigma correlator at the one-loop level. Then in Section 2.2 we consider the Linear Sigma Model (LSM) at low energies and present the $\pi \pi$ scattering amplitudes. In Section 2.3 we apply the IAM procedure to the partial wave amplitudes to recover the mass and width of the sigma. In Section 3 we analyze PA sequences at higher orders. In Section 4 the corresponding results are discussed in the context of a vector resonance model. We then conclude, in Section 5, with a short discussion.

\section{One-loop Linear Sigma Model}

\subsection{Sigma pole position up to $\mathcal{O}(g)$}

We begin presenting the results for the one-loop corrections to the mass and the width of the sigma in the LSM. At tree-level, the sigma mass is found to be $M_{\sigma}^{2}=2 \mu^{2}$, and the width is zero. At next-to-leading order, the sigma pole gets shifted due to the quartic potential, i.e., $M_{\sigma}^{2}=2 \mu^{2}+\mathcal{O}(g)$, and the width becomes different from zero.

In order to determine the scalar meson mass and width up to $\mathcal{O}(g)$, we compute the one-loop sigma correlator [3],

$$
i \Delta(s)^{-1}=s-M_{\sigma}^{2}\left[1+\frac{3 g}{16 \pi^{2}}\left(-\frac{13}{3}+\ln \frac{-s}{M_{\sigma}^{2}}+3 \rho(s) \ln \left(\frac{\rho(s)+1}{\rho(s)-1}\right)\right)+\mathcal{O}\left(g^{2}\right)\right],
$$

where $\rho(s) \equiv \sqrt{1-4 M_{\sigma}^{2} / s}$ and the term $-13 / 3$ is determined by the renormalization scheme chosen by Ref. [3], which sets the relation $2 g F^{2}=M_{\sigma}^{2}$ at the one-loop order, with $F$ the pion decay constant. Now it is possible to extract the pole $s_{p}$ of the propagator up to the considered order in perturbation theory. If one approaches the branch cut from the upper part of the complex $s$-plane, the pole in the second Riemann sheet is located at

$$
s_{p}=M_{\sigma}^{2}\left[1+\frac{3 g}{16 \pi^{2}}\left(-\frac{13}{3}+\pi \sqrt{3}-i \pi\right)+\mathcal{O}\left(g^{2}\right)\right],
$$

where we have used $s_{p}=M_{\sigma}^{2}+\mathcal{O}(g)$. The pole mass and width, defined from $s_{p}=\left(M_{p}-i \Gamma_{p} / 2\right)^{2}$, are then given by

$$
\frac{M_{p}^{2}}{M_{\sigma}^{2}}=1+\frac{3 g}{16 \pi^{2}}\left(-\frac{13}{3}+\pi \sqrt{3}\right)+\mathcal{O}\left(g^{2}\right)
$$




$$
\frac{M_{p} \Gamma_{p}}{M_{\sigma}^{2}}=\frac{3 g}{16 \pi}+\mathcal{O}\left(g^{2}\right)
$$

As expected, at lowest order the pole width agrees with that derived from the decay amplitude [14].

\subsection{Low-energy expansion}

We now consider the LSM at low energies. The contribution from the sigma exchanges to the renormalized $\mathcal{O}\left(p^{4}\right) \chi \mathrm{PT}$ couplings gives [3]

$$
\begin{aligned}
& \ell_{1}^{r}(\mu)=\frac{1}{4 g}+\frac{1}{96 \pi^{2}}\left[\ln \frac{M_{\sigma}^{2}}{\mu^{2}}-\frac{35}{6}\right]+\mathcal{O}(g), \\
& \ell_{2}^{r}(\mu)=\frac{1}{48 \pi^{2}}\left[\ln \frac{M_{\sigma}^{2}}{\mu^{2}}-\frac{11}{6}\right]+\mathcal{O}(g) .
\end{aligned}
$$

The $\pi \pi$-scattering is determined by the $\pi^{+} \pi^{-} \rightarrow \pi^{0} \pi^{0}$ amplitude, which is given up to $\mathcal{O}\left(p^{4}\right)$ in the chiral expansion by

$$
\begin{aligned}
A(s, t, u)= & \frac{s}{F^{2}}+\frac{2 s^{2}}{F^{4}} \ell_{1}^{r}+\frac{s^{2}+(t-u)^{2}}{2 F^{4}} \ell_{2}^{r} \\
& +\frac{1}{96 \pi^{2} F^{4}}\left[-3 s^{2} \ln \frac{-s}{\mu^{2}}-t(t-u) \ln \frac{-t}{\mu^{2}}-u(u-t) \ln \frac{-u}{\mu^{2}}+\frac{5 s^{2}}{2}+\frac{7(t-u)^{2}}{6}\right],
\end{aligned}
$$

where $\mu$ refers here to the arbitrary renormalization scale, and the chiral limit has been considered.

With this one constructs the definite isospin amplitudes

$$
\begin{aligned}
& T(s, t, u)^{\mathrm{I}=0}=3 A(s, t, u)+A(t, s, u)+A(u, t, s) \\
& T(s, t, u)^{\mathrm{I}=1}=A(t, s, u)-A(u, t, s) \\
& T(s, t, u)^{\mathrm{I}=2}=A(t, s, u)+A(u, t, s) .
\end{aligned}
$$

The partial wave projection is then provided by

$$
t_{J}^{I}(s)=\frac{1}{64 \pi} \int_{-1}^{1} d \cos \theta P_{J}(\cos \theta) T(s, t, u)^{\mathrm{I}},
$$

where $\theta$ is the scattering angle in the $\pi \pi$ center-of-mass rest frame.

Hence, for the first partial waves $t_{J}^{I}(s)$, with $I J=00,11,20$, one finds the following $\mathcal{O}\left(p^{2}\right)$ amplitudes,

$$
\begin{aligned}
t_{0}^{0}(s)_{(2)} & =\frac{s}{16 \pi F^{2}}, \\
t_{1}^{1}(s)_{(2)} & =\frac{s}{96 \pi F^{2}}, \\
t_{0}^{2}(s)_{(2)} & =-\frac{s}{32 \pi F^{2}},
\end{aligned}
$$


and at $\mathcal{O}\left(p^{4}\right)$,

$$
\begin{aligned}
t_{0}^{0}(s)_{(4)} & =\frac{s^{2}}{48 \pi F^{4}}\left[11 \ell_{1}^{r}+7 \ell_{2}^{r}-\frac{1}{96 \pi^{2}}\left(18 \ln \frac{-s}{\mu^{2}}+7 \ln \frac{s}{\mu^{2}}-\frac{51}{2}\right)\right], \\
t_{1}^{1}(s)_{(4)} & =\frac{s^{2}}{96 \pi F^{4}}\left[\ell_{2}^{r}-2 \ell_{1}^{r}-\frac{1}{96 \pi^{2}}\left(\ln \frac{-s}{\mu^{2}}-\ln \frac{s}{\mu^{2}}-\frac{2}{3}\right)\right], \\
t_{0}^{2}(s)_{(4)} & =\frac{s^{2}}{24 \pi F^{4}}\left[\ell_{1}^{r}+2 \ell_{2}^{r}-\frac{1}{96 \pi^{2}}\left(\frac{9}{4} \ln \frac{-s}{\mu^{2}}+\frac{11}{4} \ln \frac{s}{\mu^{2}}-\frac{51}{8}\right)\right] .
\end{aligned}
$$

For the next section, it will be suitable to rewrite the $\mathcal{O}\left(p^{4}\right)$ amplitudes in terms of the LSM parameters and the $\mathcal{O}\left(p^{2}\right)$ scattering:

$$
\begin{aligned}
& t_{0}^{0}(s)_{(4)}=t_{0}^{0}(s)_{(2)} \times \frac{11 s}{6 M_{\sigma}^{2}}\left[1-\frac{g}{264 \pi^{2}}\left(18 \ln \frac{-s}{M_{\sigma}^{2}}+7 \ln \frac{s}{M_{\sigma}^{2}}+\frac{193}{3}\right)+\mathcal{O}\left(g^{2}\right)\right], \\
& t_{1}^{1}(s)_{(4)}=t_{1}^{1}(s)_{(2)} \times\left(\frac{-s}{M_{\sigma}^{2}}\right)\left[1+\frac{g}{48 \pi^{2}}\left(\ln \frac{-s}{M_{\sigma}^{2}}-\ln \frac{s}{M_{\sigma}^{2}}-\frac{26}{3}\right)+\mathcal{O}\left(g^{2}\right)\right], \\
& t_{0}^{2}(s)_{(4)}=t_{0}^{2}(s)_{(2)} \times\left(\frac{-2 s}{3 M_{\sigma}^{2}}\right)\left[1-\frac{g}{24 \pi^{2}}\left(\frac{9}{4} \ln \frac{-s}{M_{\sigma}^{2}}+\frac{11}{4} \ln \frac{s}{M_{\sigma}^{2}}+\frac{163}{24}\right)+\mathcal{O}\left(g^{2}\right)\right],
\end{aligned}
$$

where we have used Eq. (44) and the relation $2 g F^{2}=M_{\sigma}^{2}[3]$.

\subsection{Unitarization of the $\chi \mathrm{PT}$ amplitude}

The Inverse Amplitude Method (IAM) provides an amplitude that is unitary not only at the perturbative level but exactly. This means that in the elastic limit one has for $s>0$ the partialwave relation

$$
\operatorname{Im} t(s)=|t(s)|^{2}
$$

where the indices $I J$ are assumed $\left(t=t_{J}^{I}\right)$. This relation can be reexpressed as a relation for the inverse amplitude:

$$
\operatorname{Im} t(s)^{-1}=-1
$$

Thus, the imaginary part of $t(s)^{-1}$ becomes completely determined and one only needs to specify the real part Re $t^{-1}$. The IAM relies then on a low-energy matching to $\chi \mathrm{PT}$ (with $t_{\chi \mathrm{PT}}^{-1}=$ $\left.t_{(2)}^{-1}\left[1-t_{(4)} / t_{(2)}+\ldots\right]\right)$ in order to fix the unknown part of the amplitude. Thus, at $\mathcal{O}\left(p^{4}\right)$, one has the unitarized amplitude,

$$
t_{\mathrm{IAM}}=\frac{t_{(2)}}{1-\frac{t_{(4)}}{t_{(2)}}} .
$$

This expression is sometimes also known as a $P_{1}^{1}$ Padé Approximant of the partial-wave amplitude. The IAM has been also extended up to $\mathcal{O}\left(p^{6}\right)$ by means of what is sometimes named as a $P_{2}^{1}$ approximant [10, 12]:

$$
t_{\mathrm{IAM}}=\frac{t_{(2)}}{1-\frac{t_{(4)}}{t_{(2)}}-\frac{t_{(6)}}{t_{(2)}}+\left(\frac{t_{(4)}}{t_{(2)}}\right)^{2}} .
$$


However, we want to remark that $t(s)_{\text {IAM }}$ is not a PA in the variable $s$ : It is not a rational approximant since it also contains the logarithms from the pion loops. Thus, strictly speaking no theoretical argument ensures the recovery of the physical amplitude. Only in the tree-level limit $t(s)_{\text {IAM }}$ becomes a PA. In any case, we will see that both the whole and the tree-level IAM amplitudes are unable to reproduce the original partial waves in the resonance region.

Given the $\mathcal{O}\left(p^{2}\right)$ and $\mathcal{O}\left(p^{4}\right) \chi \mathrm{PT}$ amplitudes from Eqs. (8)-(10), it is then possible to extract the poles of the corresponding $t(s)_{\text {IAM }}$ for the LSM, satisfying $1=t(s)_{(4)} / t(s)_{(2)}$ at $s=s_{p}$ :

$\mathbf{I J}=\mathbf{0 0}$

$$
s_{p}=\frac{6}{11} M_{\sigma}^{2}\left[1+\frac{g}{264 \pi^{2}}\left(\frac{193}{3}+25 \ln \frac{6}{11}-18 i \pi\right)+\mathcal{O}\left(g^{2}\right)\right],
$$

$\mathbf{I J}=\mathbf{1 1}$

$$
s_{p}=-M_{\sigma}^{2}\left[1+\frac{g}{48 \pi^{2}}\left(\frac{26}{3}+i \pi\right)+\mathcal{O}\left(g^{2}\right)\right]
$$

$\mathbf{I J}=\mathbf{2 0}$

$$
s_{p}=-\frac{3}{2} M_{\sigma}^{2}\left[1+\frac{g}{24 \pi^{2}}\left(\frac{163}{24}+5 \ln \frac{3}{2}+\frac{11 i \pi}{4}\right)+\mathcal{O}\left(g^{2}\right)\right] .
$$

These are the poles that appear in the unphysical Riemann sheet as one approaches from upper half of the first Riemann sheet. There is also a conjugate pole at $s_{p}^{*}$ if one approaches the real $s$-axis from below.

The first thing to be noticed is that poles appear in the $I J=11$ and 20 channels even for small values of $g$, contrary to what one expects in the LSM, where no meson with these quantum numbers exists. Furthermore, these "states" are not resonances, as they are located on the left-hand side of the complex $s$-plane, out of the physical Riemann sheet, and carrying a negative squared mass.

As for the $I J=00$ channel, one finds a resonance with pole mass and width,

$$
\begin{aligned}
\frac{M_{p}^{2}}{M_{\sigma}^{2}} & =\frac{6}{11}\left[1+\frac{g}{16 \pi^{2}}\left(\frac{50}{33} \ln \frac{6}{11}+\frac{386}{99}\right)+\mathcal{O}\left(g^{2}\right)\right], \\
\frac{M_{p} \Gamma_{p}}{M_{\sigma}^{2}} & =\frac{24}{121} \cdot \frac{3 g}{16 \pi}+\mathcal{O}\left(g^{2}\right) .
\end{aligned}
$$

The IAM predictions for $M_{p}^{2}$ and $M_{p} \Gamma_{p}$ result, respectively, $40 \%$ and $80 \%$ smaller than the original ones in the LSM, computed in Eq. (3). This points out the low reliability of this particular method in order to recover the hadronic properties of the theory from its effective low-energy description.

The IAM poles remain badly located even in the weakly interacting limit, so this failure cannot be attributed to non-perturbative effects. In the limit when $g \rightarrow 0$ and $M_{\sigma}$ is kept fixed one finds that the poles predicted in all the different channels fall down to the real $s$-axis. We are left with just tree-level amplitudes and the expressions become greatly simplified. Due to the smoothness of this limit, it will be assumed in the next analysis of higher order Padé Approximants $[M / N]$ and in the study of the vector model in Section 4 . 


\section{Higher order Padé Approximants for tree-level amplitudes}

In this section we consider higher order Padé Approximants to the partial wave amplitudes, with the hope that this will provide some insight on the nature of the unitarization process discussed above. We will see that the PA sequence associated with the IAM does not converge properly, and that diagonal sequences are much more suitable for this purpose. We begin with a brief overview of the theory of PAs.

\subsection{Generalities on Padé Theory}

Let $f(s)$ be a function with a Taylor expansion around $s=0$. A Pade Approximant (PA) to $f(s)$, denoted as $P_{N}^{M}(s)$, is defined as the ratio $P_{N}^{M}(s)=R_{M}(s) / Q_{N}(s)$ of two polynomials of orders $M$ and $N$, respectively, and such that $f(s)-P_{N}^{M}(s)=\mathcal{O}\left(s^{M+N+1}\right)$ when $s \rightarrow 0$.

The convergence properties of the PAs to a given function are more complex than those of the Taylor expansion. However, they converge for a broader set of functions, even in the case of slowly convergent or asymptotic power series, and they usually carry smaller errors than the Taylor expansions (when these are applicable). Furthermore, in many cases, the Padés have been found to provide a fairly good approximation even beyond their expected range of applicability.

Pommerenke's theorem states that the sequence of diagonal PAs, i.e. $[N / N]$, to a meromorphic function is convergent everywhere in any compact set of the complex plane except, at most, for a zero capacity set 1 [15, 16, 17, 18, 19, 20]. This obviously includes the poles of $f(s)$, where the original function is ill-defined. In addition, the PA may produce a series of poles absent in $f(s)$. Thus, for a given compact region $\mathcal{K}$ in the complex plane, Pommerenke's theorem of convergence requires that, either these undesired poles move away from the region $\mathcal{K}$ as the order of the PA increases, or they pair up with a close-by zero becoming what is usually called a defect [16]. Although the PA breaks down in the very neighborhood of these extraneous poles, away from them the approximation is safe. Likewise, a PA can approximate a multivaluated function (for example, a function with a logarithmic branch cut). The sequence of PAs will cluster its poles along the cut [16], as we will see in the following section.

\subsection{Tree-level PAs in the LSM}

In order to be able to handle the amplitude at higher orders, we will consider the $\pi \pi$ scattering at tree-level. This is equivalent to working in the limit $g \ll 1$ and keeping just the first non-trivial contribution in the $g$ expansion. Thus, the $\pi \pi$-scattering is determined in the LSM by the function

$$
A(s, t, u)=\frac{s}{F^{2}} \frac{M_{\sigma}^{2}}{M_{\sigma}^{2}-s},
$$

By means of the partial wave projection in Eq. (17), this provides

$$
t_{0}^{0}(s)=\frac{M_{\sigma}^{2}}{32 \pi F^{2}}\left[-5+\frac{3 M_{\sigma}^{2}}{M_{\sigma}^{2}-s}+\frac{2 M_{\sigma}^{2}}{s} \ln \left(1+\frac{s}{M_{\sigma}^{2}}\right)\right],
$$

\footnotetext{
${ }^{1}$ In addition to meromorphic, there are known theorems of convergence with PAs for Stieltjes functions, continued functions, Gauss hypergeometric functions, Bessel functions, some kind of divergent series, sets of complex points, etc. This has been applied in the past to various kinds of scattering processes [16].
} 


$$
\begin{aligned}
& t_{1}^{1}(s)=\frac{M_{\sigma}^{4}}{32 s \pi F^{2}}\left[-2+\left(\frac{2 M_{\sigma}^{2}}{s}+1\right) \ln \left(1+\frac{s}{M_{\sigma}^{2}}\right)\right], \\
& t_{0}^{2}(s)=-\frac{M_{\sigma}^{2}}{16 \pi F^{2}}\left[1-\frac{M_{\sigma}^{2}}{s} \ln \left(1+\frac{s}{M_{\sigma}^{2}}\right)\right] .
\end{aligned}
$$

The $\ln \left[1+s / M_{\sigma}^{2}\right]$ logarithms come from the partial-wave projection of the tree-level exchanges of resonances in the crossed channel. They have absolutely nothing to do with the logarithms of the $\chi \mathrm{PT}$ amplitudes in Eq. (10), which come from the $\pi \pi$ loops.

At low energies the amplitude becomes

$$
A(s, t, u)=\frac{s}{F^{2}}\left[1+\frac{s}{M_{\sigma}^{2}}+\frac{s^{2}}{M_{\sigma}^{4}}+\ldots\right],
$$

so the partial waves are given by,

$$
\begin{aligned}
& t_{0}^{0}(s)=\frac{s}{16 \pi F^{2}}\left[1+\frac{11 s}{6 M_{\sigma}^{2}}+\frac{15 s^{2}}{12 M_{\sigma}^{4}}+\ldots\right], \\
& t_{1}^{1}(s)=\frac{s}{96 \pi F^{2}}\left[1-\frac{s}{M_{\sigma}^{2}}+\frac{9 s^{2}}{10 M_{\sigma}^{4}}+\ldots\right], \\
& t_{0}^{2}(s)=-\frac{s}{32 \pi F^{2}}\left[1-\frac{2 s}{3 M_{\sigma}^{2}}+\frac{s^{2}}{2 M_{\sigma}^{4}}+\ldots\right] .
\end{aligned}
$$

The comparison between the low-energy expansions and the whole result provides a first insight of the piece of information that is lost in the unitarization procedure. At high energies, the partial waves contain poles on the right-hand side of the $s$-plane, related to $s$-channel resonance exchanges, and a left-hand cut, related to the crossed-channel resonance exchanges. At low energies, both kinds of exchanges contribute equally to the low energy couplings, so the crossed resonance exchanges shift the IAM poles from their physical value. Although $t$ and $u$ channels are not so relevant in the region close to the resonance pole, at low energies they are as important as the $s$-channel.

The simplest Padé, $P_{1}^{1}$, gives the prediction

$$
\begin{aligned}
s_{0}^{0} & =\frac{6}{11} M_{\sigma}^{2}, \\
s_{1}^{1} & =-M_{\sigma}^{2}, \\
s_{0}^{2} & =-\frac{3}{2} M_{\sigma}^{2},
\end{aligned}
$$

which agrees with the one-loop calculation from Eqs. (14)-(16) if one remains at leading order in $g$.

\subsection{Higher order PAs in the LSM}

The convergence of a sequence $[M / N]$ of Padé Approximants to a function implies that the PA tends to mimic its analytical structure as $M, N \rightarrow \infty$. The common procedure for the construction of a sequence of PAs is to increase $M, N$ following a given pattern, e.g. $M=N \rightarrow \infty$. In some cases, 

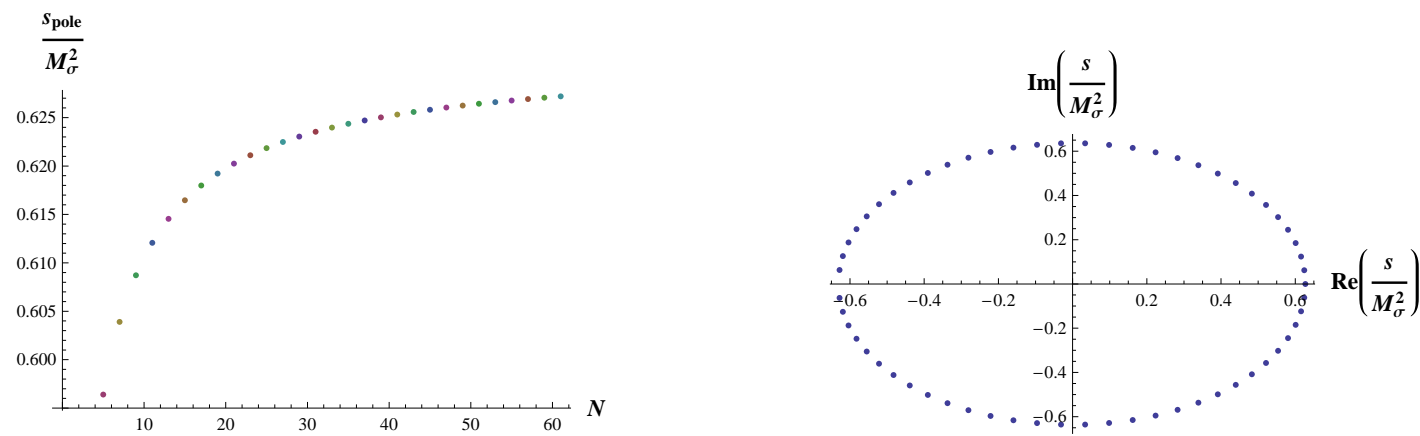

Figure 1: Left: Position of the nearest pole to $M_{\sigma}^{2}$ for the first PAs of the form $[1 / N]$ with $N$ odd (for even $N$ all the poles are complex). Right: Poles of the $P_{61}^{1}$ in the complex plane.

this allows the application of known mathematical theorems that ensure convergence [15, 16, 20]. This has been used for the study of certain Green functions [17, 18, 19. However, little is known about the sequence $[M / N]$ when $M$ is kept fixed and $N \rightarrow \infty$ (for instance $P_{1}^{1}, P_{2}^{1} \ldots$ ).

We will employ and compare the $[1 / N]$ and $[N / N]$ sequences for the study of the $\pi \pi$ partial wave scattering amplitudes. We will also comment on PAs of the $[N+K / N]$, e.g $[N-2 / N]$. In the next lines we will focus our attention on the $I J=00$ partial wave, but analogous results are found for the other channels. Former works pointed out that the PAs and other unitarizations fail to incorporate the crossed channel resonance exchanges [14, 24]. Nonetheless, we will see that as $N$ grows, the poles of the sequence $[N / N]$ actually tend to mimic not only the $s$-channel poles but also the left-hand cut contribution from diagrams with resonances in the $t$ and $u$ channels.

The sequence $[K / N]$, with $K$ fixed, is studied in the present section in the framework of the Linear Sigma Model. It provides an example of the behavior of these kind of sequences. $K=1$ is chosen because of the similarity of this sequence and the IAM [9, 10, 12, 13, 24. Our results are summarized in Fig. 1, No convergence is found with this sequence. In the case of $N$ odd, Fig. 1,a. shows that the $P_{N}^{1}$ pole closest to $M_{\sigma}^{2}$ does not approach this value even for very large $N$, always remaining a $30 \%$ below. The analytical structure of the original amplitude $(s$-channel sigma pole plus left-hand cut) is never recovered since the $[1 / N]$ PAs always set the poles in the circular pattern shown in Fig. 1,b. This suggests that the use of further $[1 / N]$ approximants to extend the IAM is not the optimal way to proceed, even if we had an accurate knowledge of the low-energy expansion up to very high orders.

Alternatively, the use of sequences such as $[N+K / N]$ (e.g. $[N-2 / N],[N-1 / N],[N / N],[N+$ $1 / N] \ldots$ ) seems to be a better strategy. In the following we analyze the sequence $[N / N]$, as it ensures the appropriate behavior at high energies, $|t(s)|<1$. Nevertheless, similar results have been generally found for the $[N+K / N]$ PAs with $K \neq 0$. The $P_{N}^{N}$ pole closest to $M_{\sigma}^{2}$ is shown in Fig. 2, a. One finds a quick convergence of the sequence: $P_{1}^{1}$ reproduces the sigma pole a $40 \%$ off but $P_{2}^{2}$ disagrees by less than $1 \%, P_{3}^{3}$ by less than $0.1 \%$, etc. Notice that already $P_{2}^{2}$ provides a much better description than $P_{61}^{1}$, although one includes far more low-energy information in the latter. All this points out the sizable discrepancy of the first element of the sequence $\left(P_{1}^{1}\right)$ with respect to the original amplitude. It also indicates that the $[1 / N]$ PAs do not produces a serious improvement. On the contrary, the $[N / N]$ sequence provide a far more efficient strategy with a quick convergence.

Likewise, Fig. 1, b shows how the $[1 / N]$ PAs are unable to recover the analytical structure of the 

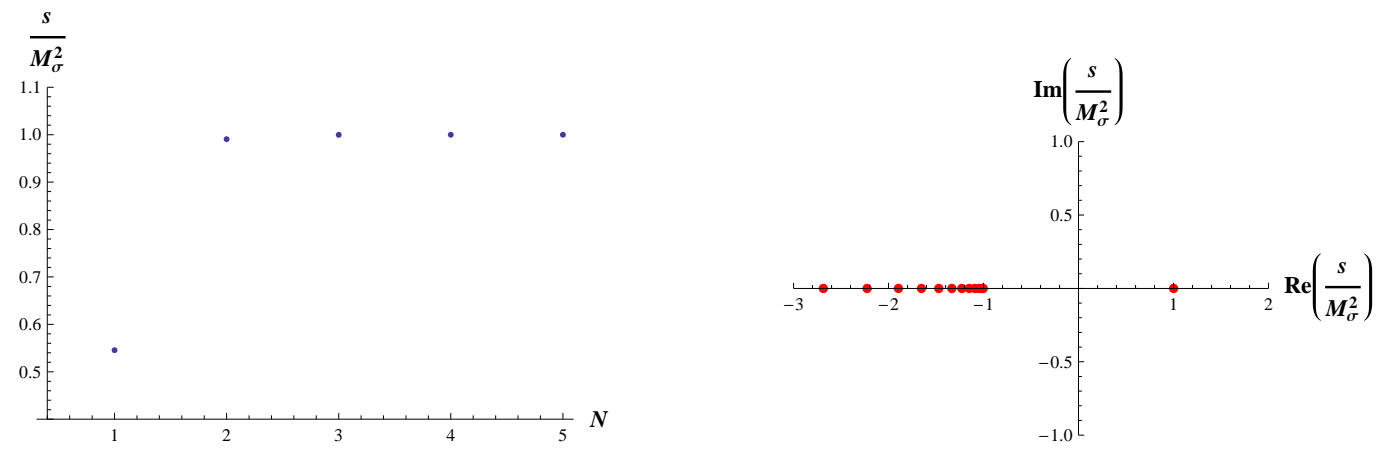

Figure 2: Left: Location of the closest pole to $M_{\sigma}^{2}$ for the first [N/N] PAs. Right: Poles of $P_{20}^{20}$.

original amplitude, whereas the $[N / N]$ sequence, besides providing the isolated pole of the sigma, tends to reproduce the left-hand cut as $N$ increases. The poles of $P_{20}^{20}$ are plotted in Fig. 2. Although a PA is a rational function without cuts, these are mimicked by placing poles where the cuts should lie. The $P_{20}^{20}$ has one isolated pole near $M_{\sigma}^{2}$ (with an accuracy of $10^{-30}$ ) and nineteen poles over the real axis at $s_{p}<-M_{\sigma}^{2}$, i.e. on the left-hand cut of the original function. As $N$ is increased, the number of poles lying on the branch cut increases too.

A remarkable feature found for the first $P_{N}^{N}$ approximants $\left(P_{1}^{1}, P_{2}^{2}, P_{3}^{3}\right)$ is that they obey exact unitarity, as it happened with the IAM sequence $[1 / N]$.

We would like to remark that, although we lack of a general theorem that ensures the convergence of the $[N+K / N]$ sequences, we have found that they reproduce the original partial waves for arbitrary $K$. Moreover, after performing modifications on the structure of the amplitudes of Eq. (19) we still found convergence the different $[N+K / N]$ PAs. In several situations the PAs set all poles over the left-hand cut position and one isolated pole that approached $M_{\sigma}^{2}$ when $N \rightarrow \infty$. In the worst cases, in addition to this we found extraneous poles that either moved away as $N$ increased or they tend to be canceled by nearby zeros at $N \rightarrow \infty$.

As an amusement, we have also probed the PA sequence $[N / 1]$ which has the same number of inputs as the $[1 / N]$ has for a given $N$. In this new case we have found convergence in both LSM and the resonance model presented in the following section but slower than the $[N / N]$. For instance, the prediction for the $M_{\sigma}^{2}$ for the first $P_{1}^{N}$ are $\frac{s_{p}}{M_{\sigma}^{2}}=0.55,1.47,0.73,1.27,0.81 \ldots$ A criticism that can be done to this sequence is its lack of unitarity, in contrast to the other studied sequences.

\section{Vector Resonance Model}

In order to broaden our analysis, we consider now a model with just vector mesons [14]. It could be derived either from the gauged chiral model [21] for the couplings $3 g_{\rho} F^{2}=M_{\rho}^{2}$, or from resonance chiral theory [22] with only vectors and the relation $3 G_{V}^{2}=F^{2}$. The $\pi \pi$-scattering is given in this model by

$$
A(s, t, u)=\frac{M_{\rho}^{2}}{3 F^{2}}\left[\frac{s-u}{M_{\rho}^{2}-t}+\frac{s-t}{M_{\rho}^{2}-u}\right] .
$$

The study of the $I J=11$ partial wave leads to the same conclusions found for the LSM. It is at first sight remarkable that, on the contrary to the previous case, one recovers $s_{p}=M_{\rho}^{2}$ from the 
first-order approximant $P_{1}^{1}$. However, the sequence $[1 / N]$ already worsens at $N=2$, where the two complex-conjugate poles are located at $s_{p}=(0.71 \pm 0.96 i) M_{\rho}^{2}$ on the physical Riemann sheet. On the other hand, $P_{N}^{N}$ exactly recovers $s_{p}=M_{\rho}^{2}$ for any odd $N$. For $N$ even, the prediction from $P_{2}^{2}$ is a $30 \%$ off, but one has again a quick convergence to $s_{p}=M_{\rho}^{2}$ as $N$ increases: $P_{4}^{4}$ disagrees by less than $0.1 \%, P_{6}^{6}$ disagrees by less than $10^{-6}$, etc.

Furthermore, the $[1 / N]$ and $[N / N]$ sequences produce, respectively, the same structure of poles found for the LSM. This is, $[1 / N]$ generates the circular structure of poles of Fig. 1.b. and the sequence $[N / N]$ places one pole at $s_{p} \simeq M_{\rho}^{2}$ and the remaining ones reproducing the left-hand cut in analogy to Fig. 2,b.

\section{Discussion}

In this letter, we have addressed the reliability of the unitarization of low-energy amplitudes through Padé Approximants. The one-loop analysis of the LSM has led to IAM predictions of the sigma mass and width respectively a factor 2 and 5 smaller than the original ones in the model.

Some disagreement has been found in phenomenological determinations of the $f_{0}(600)$ pole width [11], pointing out that the IAM is unable to recover at the same time the value for the mass and the width predicted from Roy equations [23. This discrepancy becomes even more obvious in the chiral limit, with differences much larger than the expected $\mathcal{O}\left(m_{\pi}^{2}\right)$ corrections.

It has been also shown that the $P_{1}^{1}$ mass prediction from the one-loop analysis agrees at leading order with that coming from the tree-level amplitude. This argument allowed the construction of higher order PAs based on the tree-level amplitude of the LSM and a vector model. It was found that the sequence $[1 / N]$ is unable to reproduce the original partial wave scattering amplitude whereas the PAs of the form $[N / N]$ display a quickly convergent behavior. Moreover, though we lack of convergence theorem, it was found that the $[N+K / N]$ PAs were able to reproduce the partial waves for arbitrary $K$.

For all this, we suggest the use of the $[N / N]$ sequence rather than $[1 / N]$. Unfortunately, the study on broad resonances requires to go beyond the tree-level approximation. Thus, in the real world, the $I J=00$ channel needs the inclusion of loops, precluding by now the extension to PAs beyond $\mathcal{O}\left(p^{4}\right)$ in the chiral expansion, i.e. $P_{1}^{1}$. There is also a clear limitation on our experimental knowledge of the low-energy couplings, which barely goes beyond $\mathcal{O}\left(p^{4}\right)$. However, our proposal should be still suitable for the analysis of theories with narrow resonances and a relatively good knowledge of the experimental low-energy amplitudes.

\section{Acknowledgements}

We would like to acknowledge helpful comments from S. Peris, J. Prades and H.Q. Zheng on the manuscript. We also want to thank A. Fuhrer and V. Mateu for discussions concerning the LSM. This work has been partially supported by the EU-RTN Programme, Contract No. MRTN-CT2006-035482 "Flavianet", CYCIT-FEDER.FPA2005-02211, SGR2005-00916 and by the Spanish Consolider-Ingenio 2010 Program CPAN (CSD2007-00042). 


\section{References}

[1] H. Georgi, Ann. Rev. Nucl. Part. Sci 43 (1993) 209; A. Manohar, hep-ph/9606222; A. Pich, hep-ph/9806303.

[2] S. Weinberg, Physica 96A (1979) 327.

[3] J. Gasser and H. Leutwyler, Annals Phys. 158 (1984) 142.

[4] J. Gasser and H. Leutwyler, Nucl. Phys. B 250 (1985) 465.

[5] For a review on unitarized amplitudes see J.A. Oller, E. Oset and A. Ramos, Prog. Part. Nucl. Phys. 45 (2000) 157-242.

[6] D. Atkinson, M. Harada and A.I. Sanda, Phys. Rev. D 46 (1992) 3884-3893;

D.A. Dicus and W.W. Repko, Phys. Rev. D 42 (1990) 3660-3667.

[7] J.I. Basdevant and B.W. Lee, Phys. Rev. D 2 (1970) 1680.

[8] T.N. Truong, Phys. Rev. Lett. 67 (1991) 2260-2263.

[9] A. Dobado, M.J. Herrero and T.N. Truong, Phys. Lett. B 235 (1990) 134; T. Hannah, Phys. Rev. D 52 (1995) 4971-4975; A. Dobado and J.R. Pelaez, Phys. Rev. D 47 (1993) 4883-4888; Phys. Rev. D 56 (1997) 3057-3073.

[10] T. Hannah, Phys. Rev. D 55 (1997) 5613-5626.

[11] H. Leutwyler, arXiv:0804.3182 [hep-ph].

[12] G.Y. Qin, W.Z. Deng, Z.G. Xiao and H.Q. Zheng, Phys. Lett. B 542 (2002) 89-99.

[13] J.R. Pelaez, Phys. Rev. Lett. 92 (2004) 102001; J.R. Pelaez and G. Rios, Phys. Rev. Lett. 97 (2006) 242002.

[14] Z.H. Guo, J.J. Sanz Cillero and H.Q. Zheng, JHEP 0706 (2007) 030.

[15] C. Pommerenke, Padé Approximants and convergence in capacity, J. Math. Anal. Appl., 41:775, 1975.

[16] G.A. Baker, Essentials of Padé Approximants, academic press. 1975.

[17] S. Peris, Phys. Rev. D 74 (2006) 054013, arXiv:hep-ph/0603190.

[18] P. Masjuan and S. Peris, JHEP 0705, 040 (2007), arXiv:0704.1247 [hep-ph].

[19] P. Masjuan and S. Peris, arXiv:0801.3558 [hep-ph].

[20] G.A. Baker and P. Graves-Morris, Padé Approximants, encyclopedia of mathematics and its applications, cambridge univ. press. 1996.

[21] J. F. Donoghue, C. Ramirez and G. Valencia, Phys. Rev. D 39 (1989) 1947.

[22] G. Ecker et al., Nucl. Phys. B321 (1989) 311. 
[23] I. Caprini, G. Colangelo and H. Leutwyler, Phys. Rev. Lett. 96 (2006) 132001.

[24] Z.H. Guo, L.Y. Xiao and H.Q. Zheng, Int. J. Mod. Phys. A 22 (2007) 4603-4616. 